\title{
Air-protective Cryo-FIB Tomography of Sensitive Materials for Energy Applications
}

\author{
Jiang Cui, Hongkui Zheng and Kai He \\ Clemson University, Clemson, South Carolina, United States
}

The research and development of materials for energy applications such as rechargeable batteries and catalysts heavily rely on advanced characterizations. Unfortunately, many of those materials are sensitive to air, moisture, high temperature, and electron beam, severely limiting the effectiveness of the currently available characterization methods. For example, Li metal has been widely recognized as the "holy grail" of all anode materials because of its high capacity and low voltage that can potentially be used in the nextgeneration batteries including Li-S battery and all-solid-state battery to greatly enhance their energy densities. However, Li metal is extremely sensitive to not only oxygen but also Ga-ion and electron beams, making it difficult to be characterized by techniques involving air and beam exposures (e.g., SEM, FIB, and TEM). The lack of feasible characterization methods to reveal the microstructure of $\mathrm{Li}$ is one of the main issues facing the development of Li metal anodes.

Recently, it has been discovered in several pioneer studies that the damage to the sensitive Li metal induced by electron beam or Ga-ions can be effectively alleviated by freezing Li metal down to low temperatures during the beam exposure. As a result, the morphology and crystal structure of Li were successfully probed by the FIB-SEM [1], TEM [2,3] and STEM-EELS [4] under cryogenic conditions. Despite the exciting progress, there still remains two major obstacles hindering the characterization of sensitive materials: (i) the existing cryo-FIB and cryo-EM setups mostly use conventional methods to transfer sensitive samples into the microscope columns, during which the inevitable exposure of the sample to air or liquid nitrogen will still induce damage to the sample, especially when the samples are highly reactive with oxygen or nitrogen (i.e., $\mathrm{Li}, \mathrm{Na}, \mathrm{K}$, etc.); (ii) it is relatively difficult to monitor and control the actual temperature of samples particularly in the cryo-FIB, where heat is merely drained through the sample stage.

Herein, we present a novel method to conduct the cryo-FIB tomography of sensitive materials. The sensitive materials can be prepared under the protection of Ar gas and mounted onto the FIB holder in a glove box. The tip of the holder is retractable in and out of an air-tight enclosure during the entire sample transferring process until the sample is safely released in the vacuum through a side-entry port into the FIB chamber. The holder is equipped with a precise temperature control function by coupling the liquid nitrogen cold finger with a thermal control module to maintain dynamic temperature equilibrium, and thus the sample is in excellent thermal contact with the holder so that the actual temperature of the sample can be very close to the target temperature. Using this newly established method, we have probed the interface between the $\mathrm{Li}$ metal and the $\mathrm{Li}_{7} \mathrm{La}_{3} \mathrm{Zr}_{2} \mathrm{O}_{12}$ (LLZO) solid electrolyte after cycling in a battery. Benefiting from the negligible thermal drifting and reduced damage to $\mathrm{Li}$ metal, a precise $3 \mathrm{D}$ reconstruction was performed using FIB sectioning tomography, providing abundant morphological information with a high spatial resolution, which has rarely been achieved by other conventional methods. The unique airprotective cryo-FIB method established in this study offers new opportunities for damage-free characterizations of sensitive materials and may shed lights on further understanding the working mechanisms of sensitive materials in energy-related applications. 

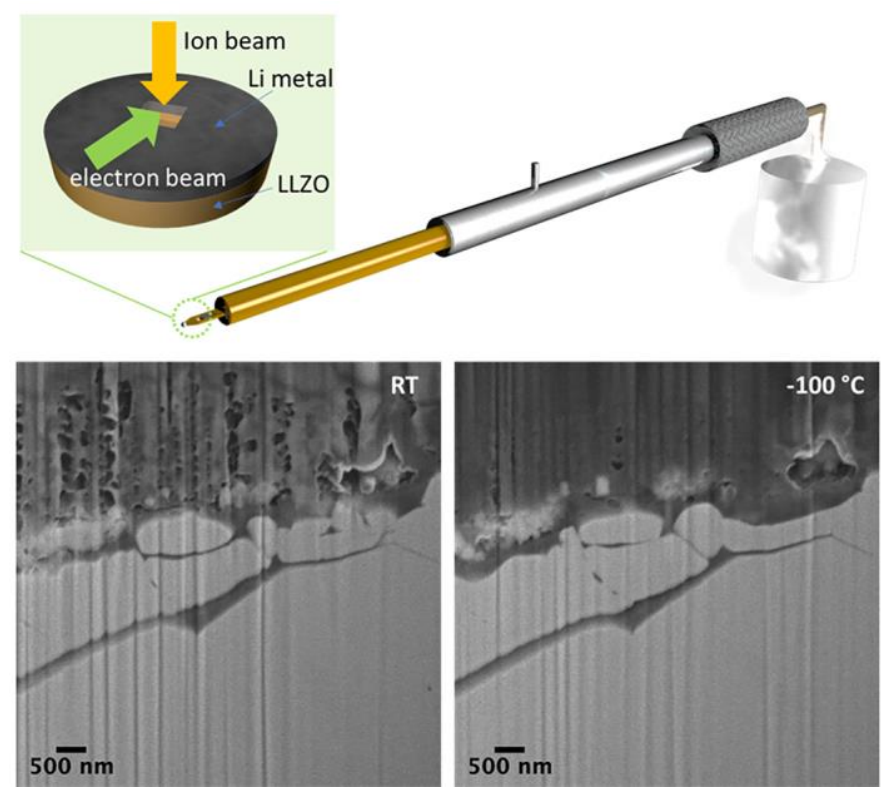

Figure 1. Schematics illustrating the air-protective cryo-FIB holder, and FIB sectioning slices of the $\mathrm{Li} / \mathrm{LLZO}$ interface under room temperature (left) and cryo-temperature (right), respectively.
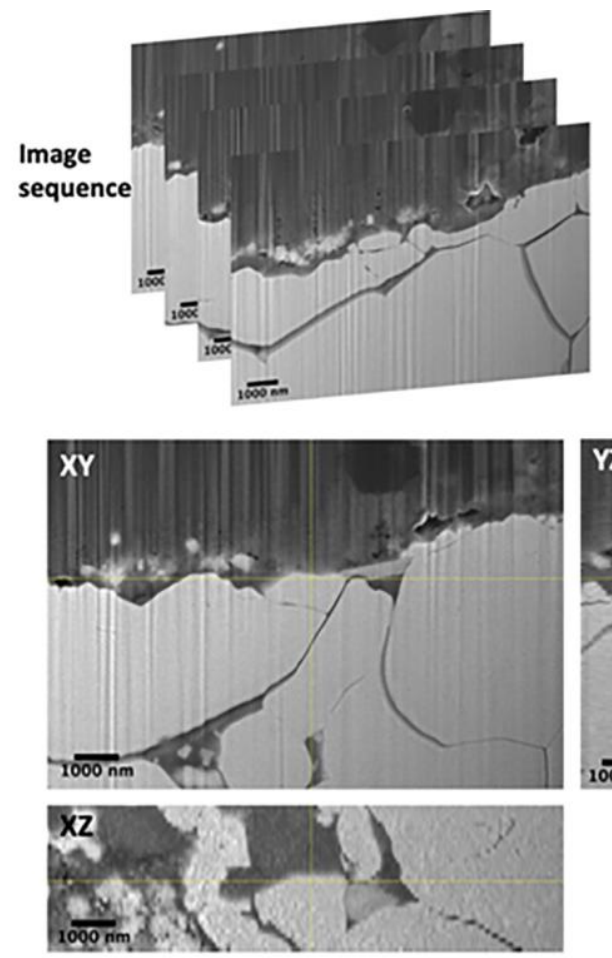
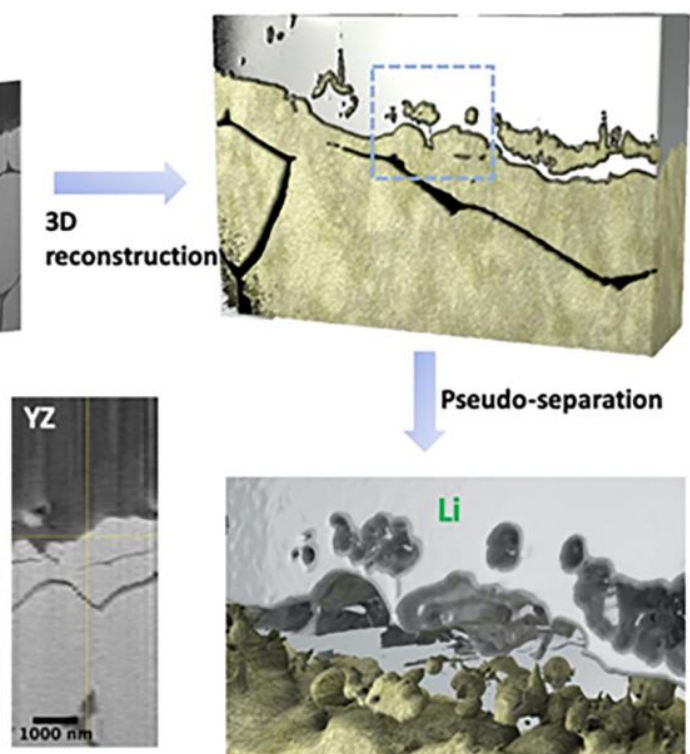

Pseudo-separation

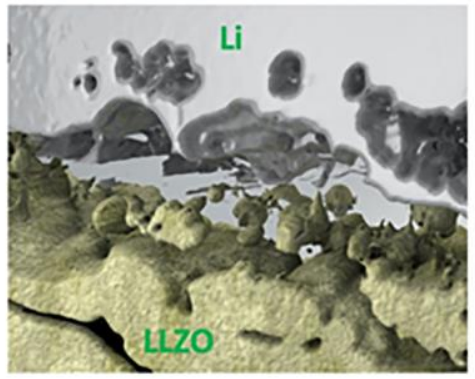

Figure 2. 3D reconstruction of the $\mathrm{Li} / \mathrm{LLZO}$ interface from FIB sectioning tomography.

\section{References}

[1] J. Z. Lee et al. ACS Energy Lett. 4, 489 (2019).

[2] Y. Li et al. Science 358, 506 (2017).

[3] X. Wang et al. Nano Lett. 17, 7606 (2017).

[4] M. J. Zachman et al. Nature 560, 345 (2018). 